\title{
Reason For Procedure
}

National Cancer Institute

\section{Source}

National Cancer Institute. Reason For Procedure. NCI Thesaurus. Code C69216.

A rationale for executing a plan of action. 